\section{Recombinant Human Soluble Thrombomodulin for Treatment of Thrombotic Microangiopathy Associated with Lupus Nephritis}

To the Editor:

Thrombotic microangiopathy (TMA) is a rare but frequently life-threatening complication of systemic lupus erythematosus (SLE). TMA is associated with a worse overall prognosis ${ }^{1,2}$ and usually requires plasma exchange in addition to immunosuppressive therapy. We treated a 33-yearold woman with SLE who developed TMA and renal dysfunction; she improved remarkably after intravenous (IV) administration of recombinant human soluble thrombomodulin (rTM) in addition to immunosuppressive therapy without plasma exchange.

The patient presented to hospital with a fever with arthralgia, digital and palmar erythema, and alopecia for 2 weeks. Laboratory findings showed pancytopenia, proteinuria with cellular casts, positive antinuclear antibodies, anti-dsDNA antibody, and anticardiolipin antibody. She was diagnosed with SLE. She was initially treated with prednisolone $15 \mathrm{mg} /$ day and aspirin $100 \mathrm{mg} /$ day. After 1 week of treatment, she was admitted to hospital because of severe headache in addition to hypertension (200/110 $\mathrm{mm} \mathrm{Hg}$ ). Her body temperature was $37.4^{\circ} \mathrm{C}$; she was conscious and alert. She had neither a stiff neck nor other neurological signs. Moist rales were heard at the lung base. Chest radiography showed cardiomegaly and lung edema.

Laboratory findings on admission are summarized in Table 1, and included proteinuria, hematuria with blood casts, poikilocytes, and elevated urinary fibrin degradation products. Complete blood counts revealed leukocytopenia, normocytic anemia with reticulocytosis, and thrombocytopenia. Other abnormal findings included hypoalbuminemia and elevated D-dimer, and low levels of haptoglobin, C3, and C4. Although direct and

Table 1. Laboratory findings on admission.

Urinalysis
Protein
Occult blood
Blood casts
Poikilocytes
Fibrin degradation products
Complete blood counts
Leukocytes
Erythrocytes
Hemoglobin
Hematocrit
Platelets
Reticulocytes
Serum analysis
Creatinine
Albumin
Lactate dehydrogenase
C3
C4
Haptoglobin
Plasma analysis
Fibrinogen
D-dimer
Coagulation
Prothrombin time
Activated partial thromboplastin time
Autoantibodies
Direct Coombs test
Indirect Coombs test
Anti-dsDNA IgG
Immune complexes (C1q-CIC)

indirect Coombs tests were negative, anti-dsDNA IgG and immune complexes were positive. She was diagnosed with nephrosis due to complicated severe lupus nephritis (LN). From the hypertension, headache, thrombocytopenia, and elevated D-dimer, malignant hypertension with possible TMA was considered.

She was immediately treated with methylprednisolone $1 \mathrm{~g}$ pulse therapy for 3 consecutive days, followed by prednisolone $60 \mathrm{mg} / \mathrm{day}$. In addition, she received nifedipine and telmisartan to treat hypertension. Despite improvement of hypertension, her serum creatinine level was further elevated to $1.05 \mathrm{mg} / \mathrm{dl}$ and thrombocytopenia progressed to $40,000 / \mu 1$ within 4 days after admission. Moreover, plasma fibrinogen level decreased to $166 \mathrm{mg} / \mathrm{dl}$. Although we speculated that her condition was either lupus-associated TMA or disseminated intravascular coagulation (DIC), a definitive diagnosis of TMA was suspended at this point because we failed to detect red cell fragmentation in the blood smears. Therefore, we started daily IV infusion of rTM $380 \mathrm{U} / \mathrm{kg} / \mathrm{day}$ on Day 4. Just after the start of rTM, her serum creatinine level improved.

As treatment for $\mathrm{LN}$, IV cyclophosphamide (CYC) was administered at $500 \mathrm{mg} /$ day $(0.9 \mathrm{mg} / \mathrm{kg}$ body weight $)$ on Day 8 . Detection of fragmented red cells on her blood smear on the following day led us to diagnose TMA associated with LN. Because of the negative Coombs test and normal prothrombin time and activated partial thromboplastin time, we excluded autoimmune hemolytic anemia and DIC. During the rTM therapy, we observed improvement of her serum creatinine, lactate dehydrogenase, fibrin degradation products, and platelet counts. Finally, on Day 18, when the disappearance of fragmented red cells was noted, the rTM infusion was discontinued. No adverse effects were noted. The prednisolone dosage was gradually tapered, and IV CYC was repeated intermittently. The proteinuria disappeared and serum creatinine level normalized. She was discharged on Day 56, and has remained in remission for more than 10 months.

The pathogenesis of TMA with SLE has not been fully elucidated. Possible explanations include malignant hypertension, antiphospholipid syndrome, thrombotic thrombocytopenic purpura/hemolytic uremic syndrome, diffuse small-vessel vasculitis, and nephritis. One hypothesis is that autoantibodies may result in a decrease in ADAMTS13 ${ }^{3}$, although some TMA patients with SLE showed normal ADAMTS13 activity ${ }^{4}$. In our case, serum ADAMTS13 was $69.8 \%$, almost within the normal range (reference range $70 \% \sim 120 \%$ ).

Current therapy for TMA with LN consists of a combination of immunosuppressive therapy and plasma exchange; there are doubts about the safety of this treatment ${ }^{5}$. Since 2008, rTM has been introduced for the treatment of DIC in Japan, based on a clinical trial ${ }^{6}$. rTM comprises the active extracellular domain of thrombomodulin, and inactivates coagulation by binding to thrombin. In addition, thrombin-rTM complexes activate protein $\mathrm{C}$. The resultant activated protein $\mathrm{C}$ inactivates factors VIIIa and V, inhibiting further thrombin formation ${ }^{7}$. Additionally, rTM has been shown to exert antiinflammatory activity by binding to and inactivating high-mobility group box 1 (HMGB1) protein. By this mechanism, rTM has been shown to inhibit endothelial injury ${ }^{8}$ and to protect against ischemic damage in the kidney ${ }^{9,10}$.

These antiinflammatory and anticoagulant effects of rTM may have contributed to the improvement of TMA with LN in our case. rTM appeared to have a therapeutic potential with fewer adverse effects compared with plasma exchange and to be useful for SLE-associated TMA. Further studies are required to confirm this.

KUMIKO TONOOKA, BSc, Assistant Professor; HIROSHI ITO, MD, Assistant Professor; TOMOHIKO SHIBATA, MD, Lecturer; SHOICHI OZAKI, MD, Professor, Division of Rheumatology and Allergology, Department of Internal Medicine, St. Marianna University School of Medicine, 2-16-1 Sugao, Miyamaeku, Kawasaki 216-8511, Japan. Address correspondence to K. Tonooka; E-mail: k.tono@f4.dion.ne.jp 


\section{REFERENCES}

1. Lansigan F, Isufi I, Tagoe CE. Microangiopathic haemolytic anemia resembling thrombotic thrombocytopenic purpura in systemic lupus erythematosus: The role of ADAMTS13.

Rheumatology 2011;50:824-9.

2. Letchumanan P, Ng HJ, Lee LH, Thumboo J. A comparison of thrombotic thrombocytopenic purpura in an inception cohort of patients with and without systemic lupus erythematosus. Rheumatology 2009;48:399-403.

3. Mannucci PM, Vanoli M, Forza I, Canciani MT, Scorza R. von Willebrand factor cleaving protease (ADAMTs-13) in 123 patients with connective tissue disease. Haematologica 2003;88:914-8.

4. Rieger M, Mannucci PM, Kremer Hovinga JA, Herzog A, Gerstenbauer G, Konetschny C, et al. ADAMTs13 autoantibodies in patients with thrombotic microangiopathies and other immunomediated diseases. Blood 2005;106:1262-7.

5. Nguyen L, Terrell DR, Duval D, Vesely SK, George JN. Complications of plasma exchange in patients treated for thrombotic thorombocytopenic purpura. IV. An additional study of 43 consecutive patients, 2005 to 2008. Transfusion 2009;49:392-4.
6. Saito H, Maruyama I, Shimazaki S, Yamamoto Y, Aikawa N, Ohno $\mathrm{R}$, et al. Efficacy and safety of recombinant human soluble thrombomodulin in disseminated intravascular coagulation: Results of phase III, randomized, double-blind clinical trial. J Thromb Haemost 2007;5:31-41.

7. Maruyama I. Recombinant thrombomodulin and activated protein $\mathrm{C}$ in the treatment of disseminated intravascular coagulation. J Thromb Haemost 1999;82:718-21 .

8. Abeyama K, Stern DM, Ito Y, Kawahara K, Yoshimoto Y, Tanaka $\mathrm{M}$, et al. The N-terminal domain of thrombomodulin sequesters high-mobility group-B1 protein, a novel anti-inflammatory mechanism. J Clin Invest 2005;115:1267-74.

9. Ikeguchi H, Maruyama S, Morita Y, Fujita Y, Kato T, Natori Y, et al. Effects of human soluble thrombomodulin on experimental glomerulonephritis. Kidney Int 2002;61:490-501.

10. Sharfuddin AA, Sandoval RM, Berg DT, McDougal GE, Campos $\mathrm{SB}$, Phillips CL, et al. Soluble thrombomodulin protects ischemic kidneys. J Am Soc Nephrol 2009;20:524-34.

J Rheumatol 2012;39:8; doi:10.3899/jrheum.111137 